\title{
Exploring the institutional logics of health professions education scholarship units
}

Lara Varpio, ${ }^{1}$ D Bridget O'Brien, ${ }^{2}$ Wendy Hu, ${ }^{3}$ Olle ten Cate, ${ }^{4}$ Steven J Durning, ${ }^{1}$ Cees van der Vleuten, ${ }^{5}$ Larry Gruppen, ${ }^{6}$ David Irby, ${ }^{2}$ Susan Humphrey-Murto ${ }^{7} \&$ Stanley J Hamstra ${ }^{8}$ (D)

CONTEXT Although health professions education scholarship units (HPESUs) share a commitment to the production and dissemination of rigorous educational practices and research, they are situated in many different contexts and have a wide range of structures and functions.

OBJECTIVES In this study, the authors explore the institutional logics common across HPESUs, and how these logics influence the organisation and activities of HPESUs.

METHODS The authors analysed interviews with HPESU leaders in Canada $(n=12)$, Australia $(n=21)$, New Zealand $(n=3)$ and the USA $(n=11)$. Using an iterative process, they engaged in inductive and deductive analyses to identify institutional logics across all participating HPESUs. They explored the contextual factors that influence how these institutional logics impact each HPESU's structure and function.
RESULTS Participants identified three institutional logics influencing the organisational structure and functions of an HPESU: (i) the logic of financial accountability; (ii) the logic of a cohesive education continuum, and (iii) the logic of academic research, service and teaching. Although most HPESUs embodied all three logics, the power of the logics varied among units. The relative power of each logic influenced leaders' decisions about how members of the unit allocate their time, and what kinds of scholarly contribution and product are valued by the HPESU.

CONCLUSIONS Identifying the configuration of these three logics within and across HPESUs provides insights into the reasons why individual units are structured and function in particular ways. Having a common language in which to discuss these logics can enhance transparency, facilitate evaluation, and help leaders select appropriate indicators of HPESU success.

\footnotetext{
${ }^{1}$ Department of Medicine, Uniformed Services University of the Health Sciences, Bethesda, Maryland, USA

${ }^{2}$ Department of Medicine, Faculty of Medicine, University of California San Francisco, San Francisco, California, USA

${ }^{3}$ Medical Education Unit, School of Medicine, Western Sydney University, Sydney, Australia

${ }^{4}$ Center for Research and Development of Education, University

Medical Centre Utrecht, Utrecht, the Netherlands

${ }^{5}$ Department of Educational Development and Research,

Maastricht University, Maastricht, the Netherlands

${ }^{6}$ Department of Learning Health Sciences, University of Michigan

Medical School, Ann Arbor, Michigan, USA
}

\footnotetext{
${ }^{7}$ Department of Medicine, Faculty of Medicine, University of Ottawa, Ottawa, Ontario, Canada

${ }^{8}$ Accreditation Council for Graduate Medical Education, Chicago, Illinois, USA
}

Correspondence: Lara Varpio, Department of Medicine, Uniformed Services University of the Health Sciences, 4301 Jones Bridge Road, Bethesda, Maryland 20814, USA. Tel: 001301295 2011;

E-mail: lara.varpio@usuhs.edu 
INTRODUCTION

As participation in health professions education scholarship (HPES) grows, ${ }^{1-4}$ individual institutions often support local engagement in this scholarship by developing health professions education scholarship units (HPESUs). ${ }^{5}$ An HPESU is an organisational structure within which a group of people is substantively engaged in HPES (Fig. 1 gives a full definition of an HPESU) ${ }^{6}$ Researchers have begun to investigate HPESUs and to describe the development of HPESUs (e.g. in departments of medical education $^{7}$ ), and activities that facilitate the success of HPESUs. ${ }^{8,9}$ Despite such interest, broadscoped international research into the organisational configurations, functions and roles of HPESUs is lacking.

During our investigations of HPESUs in Canada, Australia and New Zealand, ${ }^{9-11}$ we realised that both within and across national boundaries, individual HPESUs have very different organisational configurations and fulfil a diverse array of functions, and that the roles embedded within them vary widely. However, we also noted significant commonalities across the core values and practices of the HPESUs we studied. We were struck by this incongruity. How can HPESUs that are organised, function and staffed in such different ways still share values and practices? To investigate this incongruity, we set out to explore the underlying principles of HPESUs. We wanted to better understand the foundational values shared across HPESUs, and how they are operationalised uniquely in each local context. Turning to theories from organisational science to inform our research, we ask: Are there institutional logics that are common across HPESUs? How are these logics instantiated in each HPESU? Are there national trends for each logic?

\section{Conceptual framework: institutional logics}

Scholars in organisational science developed, investigated and refined the concept of institutional logic. ${ }^{12}$ An institutional logic is the socially constructed, historically developed pattern of beliefs and rules that shape the organising principles of an institution. ${ }^{13}$ It provides a set of norms ${ }^{14}$ for an organisation and for the individuals who work therein. Institutional logics are 'socially shared, deeply held assumptions and values that form a framework for reasoning, provide criteria for legitimacy, and help organise time and space'. ${ }^{15}$

Fields characterised by institutional complexity (e.g. health professions education [HPE]) are often comprised of institutions holding many different institutional logics. ${ }^{15-17}$ Multiple institutional logics, sometimes labelled as competing institutional logics, ${ }^{18}$ can interact in a range of ways including logic coexistence, ${ }^{19}$ the replacement of one logic by another, ${ }^{20}$ and logic blending. ${ }^{21}$ The structure and practices of an organisation reflect how different institutional logics are realised in the local context. Institutional logics, the relationships between logics, and the ways they are instantiated in an organisation constantly evolve.

To illustrate, Dunn and Jones examined the institutional logics of medical education in the USA and identified two persistent logics: the logic of care and the logic of science. ${ }^{15}$ The logic of care 'highlights physicians' clinical skills used to treat patients and improve the health of the community', whereas the logic of science 'focuses on knowledge of disease built through research and innovative treatments'. ${ }^{15}$ These competing logics have influenced medical education for decades. For instance, the authors note that, between 1947 and 1966, the budget for the National Institutes of Health $(\mathrm{NIH})$ was increased from US $\$ 8$ million to US $\$ 1$ billion. ${ }^{15}$ This period included the advent of the 1964 NIH Medical Science Training Programme, which sought to develop a cadre of physician-scientists. ${ }^{15}$ Such programmes supported the logic of science, making scientific training a critical aspect of medical student education..$^{15}$ However, at the same time, the Willard, Millis and Folsom Reports ${ }^{22-24}$ described severe shortages of primary care physicians in the USA and recommended a series of changes to health care (e.g. by calling for an individual patient's right to access to qualified physicians who will treat the patient as an individual and not as an isolated disease or organ system dysfunction). ${ }^{15}$ These calls challenged the dominant logic of science and 'advocated for a new approach and a new breed of physician, namely, family physicians, who would practise comprehensive medicine'. ${ }^{15}$ These reports put pressure on medical schools to value the logic of care. Accordingly, medical education-focused organisations were required to strike a balance between the logics of science and care. ${ }^{15}$ These logics are supported by distinct groups, fluctuate in dominance over time and shape the education of medical professionals. ${ }^{15}$ 
A health professions education scholarship unit (HPESU) is an organisational structure within which a group of people is substantively engaged in health professions education scholarship. An HPESU is often a focal point of HPES within the university and/or health centre context. An HPESU has a 'functional role' 5 at a university, college or hospital that delivers health professions education. These units may engage in the delivery and evaluation of health professions education; but to be considered an HPESU,it must include some focus on scholarship. The specific kind of organisational structure an HPESU may take varies (e.g. units, centres, departments, offices, etc.). To be recognised as an HPESU, it must meet the followingcriteria:

1 'the unit must stand asa recognisable, coherent, organisational entity in the institution', 5 AND

2 the unit must be identified as engaging in health professions education-related scholarship. That educational scholarship may be conducted at the undergraduate and/or graduate and/or continuing education levels. The unit may also house programmes that focus on teaching, service provision, professional development programme delivery, etc., but these other activities alone are not sufficient for being identified as an HPESU without the scholarship contributions.

This definition excludes units that are strictly administrative in nature or that are aimed solely at meeting educational delivery, assessment or other service needs (i.e. curriculum offices, programme evaluation offices, etc.). An HPESU may be involved in support services but, to be classified as an HPESU, there must also be production and dissemination of education-related scholarship.

We label these units as health professions (not medical) education scholarship units to include the breadth of disciplines and health professions that may be represented in the unit's membership, and in its scope of research, teaching and service work. This more inclusive scope is being adopted in several different countries, so our labelling reflects perspectives across different geographical contexts.

Figure 1 Full definition of a health professions education scholarship unit (HPESU) from Varpio $e t a l .^{6}$ HPES $=$ health professions education scholarship

In our study, we explore the institutional logics shared by HPESUs across four different countries: Canada, Australia, New Zealand and the USA. We also describe some implications of the decisions and actions HPESU leaders make when contending with multiple institutional logics.

\section{METHODS}

This study is the fourth in an international programme of research investigating HPESUs (depicted in Fig. 2). Each study was approved by the research ethics board at the relevant institution (i.e. Western Sydney University, Ottawa Hospital and the Uniformed Services University of the Health Sciences). All participants provided informed consent. Table 1 describes the participants and recruitment for each project.

The current study builds on data and analyses from Projects $1,{ }^{9} 2^{10}$ and $3^{11}$ in the programme of research. We briefly describe these projects here to give an overview of the progression of the research programme. Project 1, conducted during 20112012, was a qualitative study that used semistructured interviews to document 12 Canadian HPESU leaders' perceptions of the dimensions of unit success and of the actions commonly undertaken to achieve that success. ${ }^{9}$ Project 2, conducted during 2013-2014, was a qualitative study that used semi-structured interviews with 14 HPESU directors and 10 additional leaders from Australia and New Zealand (ANZ) regarding the structures and functions of HPESUs in ANZ and the factors that lead to unit sustainability. ${ }^{10}$ In Project 3, we reanalysed the data from Projects 1 and 2 together through recontextualisation ${ }^{25}$ to investigate how HPESU administrative leaders work as institutional entrepreneurs. $^{11}$

Project 4's research design followed a multi-step process (Fig. 3). First, while analysing the Canadian and ANZ data in Project 3, we observed the incongruity between the variety of organisational configurations, functions and roles of individual HPESUs, but also noted the values and practices common across the units. In reading theories from organisational science to inform Project 3, we came across the concept of institutional logic and felt that this concept could help us investigate that incongruity. The lead investigator (LV) began Project 4 by conducting a secondary analysis of the Canadian data, analysing the interviews by applying the concept of institutional logic to the data. By repeatedly reading and comparing ideas expressed across the dataset, LV identified institutional logics that mapped across Canadian HPESUs. 


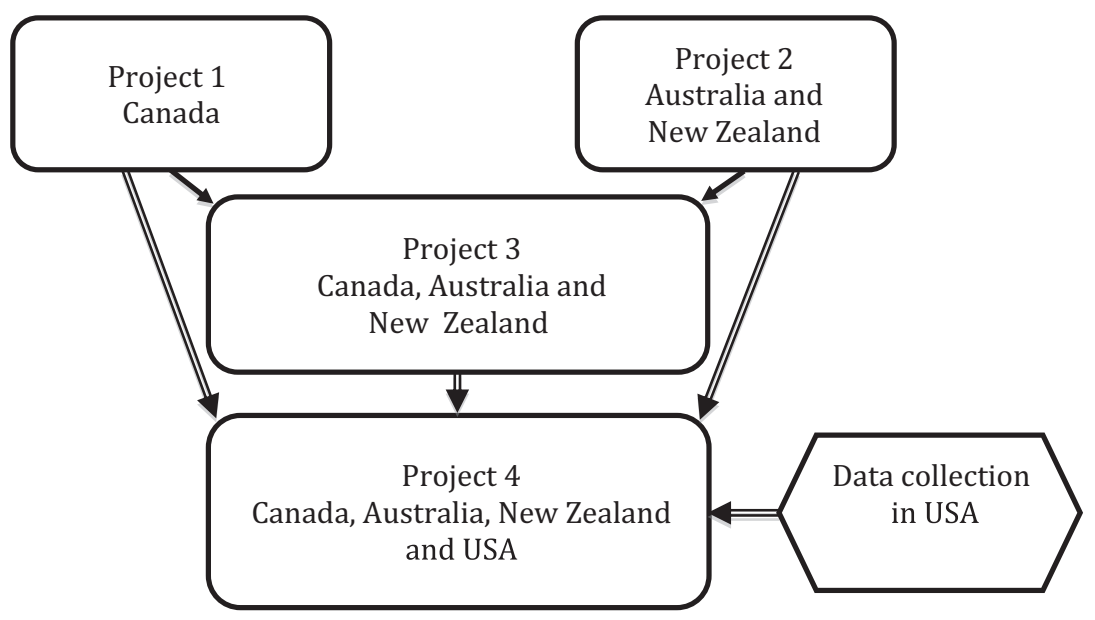

Figure 2 Four-project structure of the health professions education scholarship unit (HPESU) programme of research. Each box represents a project in the programme of research numbered according to the order in which the projects were completed. Single-line arrows indicate how data from Projects $1^{9}$ and $2^{10}$ were analysed together for Project $3 .^{11}$ Double-line arrows indicate how US data and data and analyses from Projects $1,{ }^{9} 2^{10}$ and $3^{11}$ were combined into Project 4 (reported herein)

Next, two investigators ( $\mathrm{LV}$ and $\mathrm{BO}$ 'B) engaged in a secondary analysis of the anonymised ANZ transcripts using the concept of institutional logics to inform analysis. By reading and making comparisons across the ANZ and Canadian datasets, these investigators vetted and revised the list of institutional logics developed in Step 1. Given our interest on the institutional logics that were common across HPESUs, the list was revised to include only those that applied to both the Canadian and ANZ contexts.

Meanwhile, the research team launched a study exploring US HPESU leaders' perceptions of the dimensions of success for HPESUs and the factors that enable or impede the attainment of that success. Using maximum variation sampling, ${ }^{26}$ we recruited six American HPESU leaders (Table 1) to be interviewed for this study. From April 2015 to September 2015, the study's research assistant conducted telephone interviews, lasting 41 minutes to 55 minutes, using a semi-structured interview protocol derived from the protocol used in the Canadian data collection process and revised with items from the ANZ interview protocol.

(Appendix S1 [online] provides an abbreviated version of that protocol.) This protocol was reviewed, revised and then approved by the entire research team. Two researchers ( $\mathrm{LV}$ and $\mathrm{BO}$ 'B) inductively analysed the anonymised transcripts to construct understandings of the data and develop themes for coding the data. These two researchers also deductively applied the concept of institutional logics to these developing understandings and themes to explore whether or not the concept augmented their interpretations. Analysis continued via increasing levels of abstraction to identify the practices, assumptions, values, beliefs and rules that we interpreted as constituting the institutional logics of US-based HPESUs.

These researchers then refined the application of institutional logic to the totality of our international datasets. The same two researchers (LV and BO'B) revised the developing understanding of the institutional logics shared across HPESUs to accommodate the American data while remaining true to the Canadian and ANZ findings. The researchers engaged in reflexive dialogue about the qualities of HPESUs that were unique to the USA, and those held in common with the Canadian and ANZ contexts. From December 2015 to February 2016, LV and BO'B revised the interview protocol used with US HPESU leaders to reflect the growing insights into the contexts and practices of American HPESUs, as well as the developing understanding of the institutional logics that mapped internationally across HPESUs. The full research team reviewed, revised and approved this interview protocol in early March 2016. Telephone interviews with an additional five American HPESU leaders were conducted using the new interview protocol from late March 2016 to May 2016. LV and BO'B analysed these interviews, which ranged from 49 minutes to 92 minutes in length. The full 
Table 1 Participants in each project in the programme of research

\begin{tabular}{|c|c|c|}
\hline Canada $^{9}$ & Australia and New Zealand ${ }^{10}$ & USA \\
\hline $\begin{array}{l}\text { The directors of all } 16 \text { HPESUs in } \\
\text { Canada were approached for } \\
\text { participation. Three directors declined. } \\
\text { Two sites asked that two individuals } \\
\text { participate as leadership of the HPESU } \\
\text { was shared. The unit in which the } \\
\text { principal investigator and collaborating } \\
\text { investigators worked did not } \\
\text { participate. In total, } 13 \text { interviews were } \\
\text { conducted with } 14 \text { participants from } \\
12 \text { Canadian HPESUs }\end{array}$ & $\begin{array}{l}\text { Medical education leaders from Australia's } 19 \\
\text { and New Zealand's two medical schools were } \\
\text { invited to participate. In total, } 24 \text { leaders } \\
\text { were interviewed for this study ( } 21 \text { from } \\
\text { Australia and three from New Zealand). } \\
\text { Fourteen participants were HPESU directors, } \\
\text { and } 10 \text { additional participants were leaders } \\
\text { from across ANZ. Data from both countries } \\
\text { were analysed together because the number } \\
\text { of New Zealand HPESUs is small, and these } \\
\text { countries share common accreditation and a } \\
\text { joint professional organisation for deans and } \\
\text { medical schools }\end{array}$ & $\begin{array}{l}\text { Aiming for maximum variation, we } \\
\text { recruited HPESU leaders from across the } \\
\text { Group on Educational Affairs regions of } \\
\text { the Association of American Medical } \\
\text { Colleges. We recruited HPESU leaders } \\
\text { from newly developed through to } \\
\text { long-established HPESUs, and from units } \\
\text { with wide-ranging research outputs (i.e. } \\
\text { HPESUs with high to low numbers of } \\
\text { peer-reviewed publications per year). } \\
\text { Twelve leaders were approached and } \\
11 \text { consented to participate in the study }\end{array}$ \\
\hline
\end{tabular}

ANZ = Australia and New Zealand; HPESU = health professions education scholarship unit.

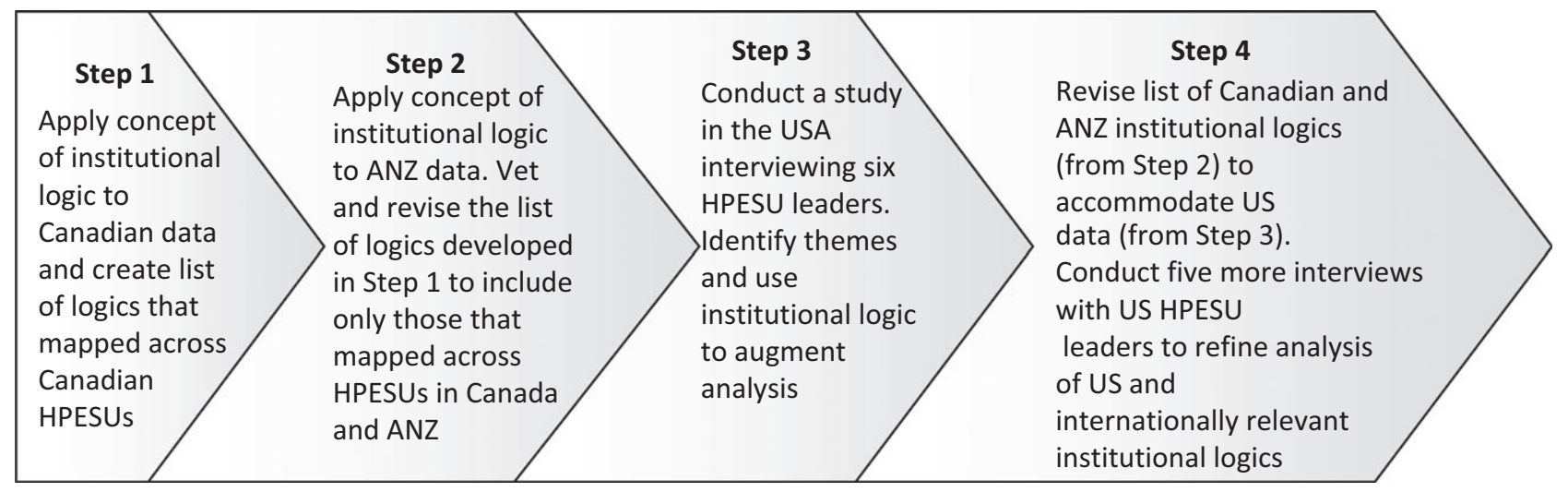

Figure 3 Multi-step research process used in Project 4. HPESU = health professions education scholarship unit; ANZ $=$ Australia and New Zealand

research team reviewed and revised the developing understanding of institutional logics common to HPESUs in Canada, ANZ and the USA. From April 2016 to August 2016, the entire research team debated, amended and finally confirmed the analyses of all the international data.

This approach to data collection and analysis enabled our research team to engage in an interpretive process of constructing insights by actively and purposefully interacting with the study participants, and by working as a research team to discern patterns across the datasets. We acknowledge that background information about the members of our research team is as important as background information about the participants we interviewed. Our team consists of three individuals who are trained and practise as physicians (WH, SJD and SH-M). Nine of our team members hold $\mathrm{PhDs}$ (in education: BO'B, WH, OtC, SJD, CvdV, DI; in English and rhetoric: LV; in psychology: SJH, LG; in medicine: WH). Several of our team members are currently or have served as HPESU leaders (WH, OtC, SJD, SJH, CvdV, LG, DI, SH-M). All team members have worked or are currently working in an HPESU. Our team 
members also come from several different national backgrounds, including Canada (SH-M, LV, SJH [note that in the past 5 years, $\mathrm{LV}$ and $\mathrm{SJH}$ have moved to work in the USA]), the USA (BO'B, DI, LG, SJD), Australia (WH), and the Netherlands $(\mathrm{OtC}, \mathrm{CvdV})$. In analysis discussions, team members often drew upon their national-level knowledge, and on their experiences of leading or working in different HPESUs. The broad range of experiences in our team supported us in identifying institutional logics that were present across all the nations represented in this study and, when possible, in identifying whether there were national-level commonalities in how those logics were manifested in HPESUs.

\section{RESULTS}

Across the Canadian, ANZ and US data, individual leaders described how their local HPESUs were shaped by fundamental principles that imposed practices, assumptions, values, beliefs and rules onto the HPESU. In other words, HPESU leaders articulated three institutional logics that influenced the HPESU's organisational structure, functions and measures of success. These were: (i) the logic of financial accountability; (ii) the logic of a cohesive education continuum, and (iii) the logic of academic research, service and teaching. We explain each of these logics below and describe nationallevel trends where these were identifiable. Table 2 illustrates these logics with data excerpts.

\section{The logic of financial accountability}

The logic of financial accountability was a driving force behind many organisational decisions taken and practices adopted by HPESU leaders. The logic of financial accountability drove HPESU leaders to focus resources in specific ways. Despite its palpable influence in each context, we did not discern consistent national-level trends for this logic; instead, local contextual factors (e.g. the history of education research, the current dean's perspective on the value and role of education scholarship, individual scholars' ability to secure external grant funding, etc.) exerted significant influence on how this logic was manifested in each HPESU (see Table 2 for data excerpts).

For example, in some contexts, leaders described focusing their unit's efforts narrowly on medical education (e.g., studying and/or delivering education programmes for medical learners).
Although these HPESU leaders often expressed a desire to engage with all of the health professions, their scope was limited by the responsibility to offer a return on the financial investment of the unit's main financial supporter, usually the local medical school or the local hospital's clinical departments. In other contexts, in which the logic of financial accountability was less pervasive or financial support was shared across many health professions (e.g. in contexts in which medicine, dentistry and nursing all contributed towards the funding of the HPESU), leaders could focus more broadly on HPE. The scope of the HPESU's work depended largely on the conditions of the unit's financial support.

The power of the logic of financial accountability to shape the scope and direction of HPESU work is formidable, pervading many aspects of each HPESU. Some HPESUs relied significantly or completely on 'soft' funds. These funds could be allocated on a non-repeating or annual basis by the medical school or the hospital's clinical departments, or might represent funds generated by the winning of external grants. In such a situation, the HPESU often had to demonstrate to its financial supporters that the HPESU's activities directly benefited those supporters' interests. By contrast, other HPESUs relied on 'hard' funds (e.g. were designated as departments in the university or hospital, and so enjoyed the financial security of being funded by a recurring institutional budget line). These HPESUs might enjoy relative financial independence and so had different development opportunities.

It is important to note that the logic of financial accountability manifested differently in individual contexts. For instance, in some contexts financial independence (e.g. having hard funding) enabled HPESUs to work across the health professions or the education continuum. However, in other contexts, hard funding was granted to the HPESU in return for specific kinds of work (e.g. servicing the undergraduate medical curriculum). Indeed, the impact of the logic of financial responsibility was exhibited uniquely in each context. However, the influence of the logic of financial accountability was always present, prompting HPESU leaders to repeatedly describe being either free from or constrained by this logic.

\section{The logic of a cohesive education continuum}

Another logic with which HPESU leaders were required to contend was the logic of medical 
Table 2 Data excerpts illustrating each institutional logic

\section{Institutional logic: logic of financial accountability}

With respect to the HPESU's focus on medicine or on health professions

'When I was hired nearly 8 years ago, I was hired to work at the medical centre level and the intent was for me to support scholarship or evaluation or design in each of the four schools we have in our medical centre. So that's medicine, dentistry, public health and nursing. But a couple of years into my tenure, they said that other schools weren't providing funding and so I was told that my scope was narrowed to just the medical school" (USA, Part 2)

"On paper, officially, we [the HPESU's members] are supposed to involve all health professions... but, in fact, for [name of HPESU] we are mainly involved with medical programmes. It's not because we don't want to be involved with other health science programmes, but it's a question of who provides our resources" (Canada, Part 14)

"I suppose the only thing we haven't really discussed is the notion of generic health professional education...I think this is an interesting area where I think many of the successful medical education units are fitting within medical schools. And I think a lot of that is around the fact that there is funding - sustained funding" (ANZ, Part 107)

\section{Institutional logic: logic of a cohesive education continuum}

"We [members of the HPESU] work with everyone and for everyone in the Faculty of Medicine which includes UGME, PGME and CME" (Canada, Part 9)

"We build capacity for innovation in research, and we do it across the continuum of undergrad, post-grads and CPD [CME]" (Canada, Part 1)

"We engage daily with pre-admissions, admissions, students' programmes, undergraduate medical education and assessment. We do not engage on a routine basis with graduate medical education. They're organised in a different space and so we will occasionally consult with them but that is very infrequent. CME we will consult on a specific programmatic evaluation or if we're sponsoring a faculty development course and we need CME credits but again that's fairly infrequent. So $98 \%$ of our interaction will be in undergraduate medical education" (USA, Part 9)

"The GME is functioning on its own, and they're getting their own educational researcher and so I've disassociated [name of the HPESU] from that for the most part" (USA, Part 5)

"The health services here do not have a history, a cultural history, of supporting learning and teaching or indeed [education] research. . . You do still hear, in this part of the world, people say: "I'm a clinician. I don't teach." For me, that's just illogical nonsense in the 21st century. It doesn't make sense. But people view them [health care delivery and physician training] as separate enterprises" (ANZ, Part 137)

\section{Institutional logic: logic of academic research, service and teaching}

\section{Labelling of the HPESU as a research unit}

"The majority of our work is applied research done in the context of the educational mission here at the medical school" (USA, Part 1)

"Our mandate is to promote and foster educational scholarship, also research development in medical education" (Canada, Part 12)

"In the last 2 to 3 years, we have shifted the focus of those academics in the unit to now start to broaden their academic activities and get more involved in research" (ANZ, Part 109)

Labelling of the HPESU as a service unit

"Our unit is primarily a service unit" (Canada, Part 13)

"We are a quality improvement shop" (USA, Part 9)

"The [HPESU] is largely responsible for the operational delivery of the programme" (ANZ, Part 123)

Service units avoid focusing on research...

"[l am] advocating that we stay aligned with the core mission and not get sucked into grants that then fragment us and distract us from what we're supposed to be doing" (USA, Part 2)

"I have often said we don't need any more PhD tenure track faculty positions in our unit. We need more Masters and PhD-trained people who are not faculty and are not tenure track, and who can dedicate themselves almost entirely to our services instead of pushing out papers" (Canada, D13)

"It was thought from the outset, when the medical school was established, that you wouldn't be a credible medical school without a standalone medical education unit that could contribute the educational expertise into the development and maintenance and innovation of the curriculum, and the assessment programme, and the evaluation of continuous quality improvement of that 
Table 2 (Continued)

programme. That was the rationale of having an [HPESU] at the outset and fundamentally that rationale continues unchanged until this day" (ANZ, Part 105)

... but even service units recognise research and teaching as part of the HPESU's required work activities

"We also then want to build on that service activity so I guess another marker [of success] would be how many dissemination products have we produced? [...] and that then blurs the distinction between what you may have been referring to as research and what we see as the service or the actual teaching function" (USA, Part 2)

"Our unit is a service unit and we're dedicated to supporting the educational mission of [the university]... And then we also provide support for students, faculty, residents who are interested in research or evaluation or a scholarly project in education... Because our unit is a service unit, it [education-related research] is not for the benefit of the members that are in our unit [the PhD-trained scientists]. Our unit exists to help other people be more successful in that [research]" (Canada, D13)

"The third area [of HPESU focus, after curriculum delivery and assessment/evaluation work] is what I would call the scholarship of teaching and learning, and this is where those in the medical education unit, not exclusively, often in conjunction with people who are working in clinical roles or other roles in the medical school, actually engage with a research agenda around the scholarship of teaching and learning and that we make contributions nationally and internationally to that literature" (ANZ, Part 105)

Some HPESUs focused on interrelationships between service, teaching and research work

"We try very, very hard not to foster a split between those [research, teaching, and service work]" (Canada, Part 3)

"I work very hard to not distinguish between them [research, teaching and service work]" (USA, Part 7)

"It's [medical education] not a purely theoretical discipline. It has to have a connection with clinical work and clinicians in order to keep its relevance and its meaningfulness... If you're doing only research and not the translation, and if you're doing only the chores, you're doing things that everybody could do" (ANZ, Part 116)

Directors who highlighted the interrelations between service, teaching and research work used specific strategies to build those connections

One director explained how service work ('The dean called me into his office and said, "Well, it's time we changed the medical curriculum. We've been resting on our laurels... Design me the ideal medical school."' [Canada, D2]) was harnessed as a research opportunity ('It also exemplified the way I like to do business which is you mount an innovation but you mount it in parallel with a research programme, and then you don't proceed along the innovation unless you have good research evidence that it's doing what you expect it to do' [Canada, D2]), and as an opportunity to mentor a clinician (be it a medical student, resident or staff physician) to be an education researcher ('We mentor individuals [individual clinicians] ... everything from the formulating of the research question to doing the [data] analysis to writing the papers' [Canada, D2])

Leaders also described how fulfilling service commitments could easily eclipse research expectations

"Right now everyone on the team does both [service and research work]. What I call feeding the monster [service work]: we need to make sure that things continue to run. If we could carve out time, and say $X$ percent of your time is going to be spent on advancing the scholarship mission, I think that would be helpful. Helpful for me personally, in my own work, and I think it would be helpful for everybody on the [HPESU] team. But that's just not the reality of where we live. I think that the downside to that is the real synergy that we experience between doing the actual authentic work and then studying that in a rigorous way - that has proven to be the case over multiple projects - allowed us to actively engage people that we probably wouldn't get to, clinical educators that we probably wouldn't get to. So, I want to watch that balance pretty carefully. I don't think I would want a scholarship team and a say, you know, a feed-the-monster team. I think there has got to be some integration there. But we continue to be challenged with what needs to be done every day getting in the way of as much scholarship as we'd like to do" (USA, Part 9)

ANZ = Australia and New Zealand; CME = continuing medical education; CPD = continuing professional development; $\mathrm{D}=$ director; $\mathrm{GME}$ = graduate medical education; HPESU = health professions education scholarship unit; Part = participant; PGME = postgraduate medical education; UGME = undergraduate medical education.

education as a continuum. Leaders described how, in some contexts, the HPESU's work with undergraduate (UME), graduate (GME) and continuing medical education (CME) was seen to represent a coherent continuum, whereas in others this work was conceived of as comprising three 
separate elements (see Table 2 for data excerpts). This logic deeply influenced the priorities of HPESU activities. We noted that this logic was realised differently in different countries.

In Canada, HPESU leaders almost universally described being able to attend to any or all aspects of the medical education continuum. The Canadian HPESU leaders did not highlight the distinctions dividing UME, GME and CME; instead, they emphasised how these elements were connected across a learning continuum. This logic is embodied in the structures of Canadian medical education. For instance, in Canada, each medical school exists as part of a university. Each Canadian GME programme is part of the same university that houses the medical school. Thus UME and GME are connected by strong organisational links. Further, the Royal College of Physicians and Surgeons of Canada, which accredits the residency programmes at the 17 universities across Canada, also accredits the learning activities of physicians in practice for continuing professional development and runs a maintenance of certification programme to meet the lifelong learning needs of Canadian physicians. This supports strong organisational connections between UME, GME and CME in Canada. This inter-connection is often reflected in how HPESUs direct their efforts across the medical education continuum.

In the USA, HPESU leaders regularly expressed interest in working across the continuum. However, many leaders focused their efforts on UME. In the US context, structural divisions between UME, GME and CME could significantly impact on the work of the HPESUs. As US GME programmes are often housed within hospitals that are not organisationally connected to medical schools, there is often an organisation-level separation between GME and UME. Given that US HPESUs are often housed in the medical school, this organisational separation can impede HPESU members from working on GME projects. Although that barrier was never described as insurmountable, it was frequently acknowledged as obstructing GME engagement.

In ANZ, the system-level differences mirror those of the USA. Moreover, the ways by which UME and GME are funded and delivered, whereby multiple education and training providers compete for education and training places in the same locations, have fostered HPESUs that are largely housed in university medical schools and focused on UME. Graduate medical education activities, largely delivered and embedded in health services, are separate and distinct from the HPESU's UME focus. Given these organisational divides, education research and GME teaching activities are often seen as secondary considerations for ANZ HPESU leaders.

\section{The logic of academic research, service and teaching}

Another important logic identified by HPESU leaders was the logic of academic research, service and teaching (see Table 2 for data excerpts). In analysing our data for this logic, we did not discern clear national-level trends. Instead, this logic manifested uniquely in each HPESU.

Leaders of HPESUs described requirements for unit members to attend to a wide range of service needs. These included, but were not limited to: sitting on various institutional committees; engaging in programme evaluation/accreditation support work; working with educators to help them become more effective in the classroom; analysing curriculum outcomes and learner experiences; and taking on administrative responsibilities for different aspects of the institution's work (e.g. leading the promotion and tenure committee). The marker of success chiefly associated with this service work was client satisfaction. This might be indicated by, for example, leadership satisfaction with the work of the HPESU, stakeholder perceptions of the HPESU as offering a valuable return on investment, continued funding from stakeholders, faculty members feeling they are getting the help they need to be effective educators, the maintenance of accreditation, the creation of reports of student assessment or programme evaluation data that university leadership and department leaders deem to be informative and actionable, among other indicators.

Leaders of HPESUs also clearly identified a need to engage in education research. Most HPESU leaders described this research in very broad terms, inclusive of the scholarships of discovery, integration, application and teaching (as defined by Boyer $^{27}$ ). Most HPESU leaders used the term 'research' to encompass all these forms of scholarship. To reflect the terms used by our participants, we refer to work in all these areas as 'research'. Associated markers of research success included publication in peer-reviewed research journals, grant capture, dissemination of findings at national or international academic conferences, 
uptake of locally developed educational innovations in other contexts, and so forth.

The teaching work that HPESU leaders described included, for example, teaching in faculty development activities and teaching courses (e.g. as part of the medical school/UME curriculum, or graduate courses for health professional degree programmes, etc.), with markers of success being associated with positive teaching evaluations. Leaders of HPESUs also noted that teaching might take the form of mentoring individual clinician-educators to engage in education scholarship. This often involved one-on-one collaborations between HPESU members and clinician-educators, and might also involve mentoring clinician-educators through graduate programmes in HPE or medical education, in which HPESU members act as faculty instructors. This mentoring was described as particularly important to the success of the HPESU as it created a community of HPES scholars who recognised the value of the HPESU and could take leadership roles in the local medical school or teaching hospital. Markers of success for this kind of teaching included capacity building (e.g. increased numbers of clinician-educators becoming active education scholars and leaders in local UME, GME or CME activities) and, when relevant, the completion of graduate degrees by clinician-educators.

Although all participants acknowledged the local institution's need for work in these three areas, each HPESU had a unique configuration of service, research and teaching engagement. Some leaders quite clearly labelled their HPESU as a service unit, whereas others emphatically described the HPESU as a research unit. Primarily in the ANZ context, some HPESU leaders defined their unit as having a teaching focus.

Leaders described service, research and teaching elements as interrelated, regardless of how the leader labelled the HPESU's focus. What varied was the relative strength of those interrelations. Some directors described the connections between service, research and teaching work as being loosely held. For instance, service-oriented HPESU leaders described avoiding focusing on research-related markers of success so that the unit could remain directed towards its service mission. However, even in these service units, research and teaching expectations were seen as related to service efforts. Other leaders explicitly stated that service, research and teaching elements were so intimately connected that they actively tried not to distinguish among them at all.

To summarise, the logic of academic research, service and teaching was a dominant logic that grounded the work of the HPESUs. In each HPESU, the three elements exist to various degrees, coexist interdependently, and display interrelationships of varying strength.

\section{How competition among these three logics is enacted in individual HPESUs}

In different contexts, each logic may hold different levels of influence and power over the HPESU. For instance, when the logic of financial accountability holds sway, the HPESU will tend to invest its efforts in supporting the needs, expectations and desires of funders. Thus, if the funding of an HPESU comes primarily from an undergraduate-focused medical school, the HPESU's activities will be largely oriented towards UME. The logic of the medical education continuum will be eclipsed, and interprofessional engagement will be limited. The HPESU's service, research and teaching activities will be oriented towards the needs of the UME funder.

In contexts in which financial accountability is not the dominant logic, the HPESU's activities can be oriented across the health professions, across the medical education continuum, and across research, teaching and service activities. In this situation, other factors (such as the interests and skills of HPES research scientists and clinician-educators, the availability of additional grant funding, the opportunity to study educational innovations, etc.) direct the HPESU's activities.

\section{DISCUSSION}

This study explores how HPESUs can share similar institutional logics that take on very different forms when instantiated in different organisations and in different countries. We identified three institutional logics that HPESU leaders engaged with and interpreted to run their HPESU: (i) the logic of financial accountability; (ii) the logic of a cohesive education continuum, and (iii) the logic of academic research, service and teaching.

The fact that these logics were pervasive across our interviews with HPESU leaders from around the world may be unsurprising because these 
logics are deeply embedded social constructs with deep historical roots. They are patterns of beliefs and rules that are foundational to the health professions. Individuals are exposed to and learn institutional logics through their education and work experiences. ${ }^{28}$ For many HPE community members, training to be a physician was an introduction to these institutional logics. Working with, within or as a leader of an HPESU further exposed individuals to these logics, shaping their practices, interests and identities. ${ }^{29}$

Although these logics have considerable influence on individuals, it is important to note that individuals can exercise agency in determining how institutional logics will guide the activities of an HPESU. Institutional logics influence the cognition and actions of the individuals who work within them, but, in turn, those individuals 'can influence how logics are instantiated in organisations'. ${ }^{14}$ Institutional logics can be conceived of as offering broad sets of cultural justifications, ${ }^{14}$ or as tools that can be 'continuously combined, configured, and manipulated to serve the purposes of actors'. ${ }^{19}$ Thus, there is a mutually constituting relationship between institutional logics and an individual's actions: 'institutional logics shape rational, mindful behaviour, and individual and organisational actors have some hand in shaping and changing institutional logics. ${ }^{18}$ By understanding HPESUs as manifestations of institutional logics, individuals can develop strategies for creating, maintaining or reconfiguring an HPESU to be both locally and externally successful. This dual focus also helps to explain why HPESUs can commonly embody these three logics, but manifest each of the logics in different ways.

The pervasive and often implicitly felt power of these institutional logics should not be underestimated. Our research team has had to contend with them during the course of this programme of research. For instance, in developing the definition of HPESUs, our team regularly debated whether the units we were studying should be labelled as health professions education scholarship units or medical education scholarship units. ${ }^{6}$ By framing these discussions in terms of competing institutional logics, we can better understand why our debate was not completely resolved. In contexts in which the logic of financial accountability is powerful and in which funding comes exclusively from medicine, the idea of labelling these units as health professions-oriented is counter-intuitive. We imagine that many readers will conceive of their local unit as a medical education scholarship unit and not as an HPESU because the logic of financial accountability is a dominant force.

Examining the institutional logics embodied in HPESUs can help us consider the possible problems an individual unit might face. For instance, consider a US HPESU that: (i) is dominated by the logic of financial accountability (with financial support coming from the medical school); (ii) is housed in the medical school and has very weak connections to UME and GME, and (iii) heavily prioritises academic service over teaching and research activities. This HPESU will often have one or several PhD-trained HPES research scientists on staff. The work of the scientists in this HPESU will be significantly directed towards successful engagement in UME-oriented service work. However, for many of these HPES research scientists, promotion in the university is based on research productivity. This situation places the research scientist between conflicting logics. To be successful in the HPESU, the scientist must engage in service activities. However, to progress through the university's academic ranks, that same scientist should focus on research productivity. This tension can make hiring and guiding highly skilled individuals into HPESU research scientist roles challenging.

As this example suggests, understanding the institutional logics embodied in an HPESU should inform the measures of success the unit is expected to meet. Our research participants commonly identified academic research as the criterion of achievement by which they are measured and by which they measure other HPESUs. However, many of these same participants led service-oriented HPESUs, or at least HPESUs in which research was not the dominant element in the logic of academic research, service and teaching. An HPESU that is driven by logics of financial accountability and academic service should not be primarily evaluated by the number of research papers published by its members because this marker of success is not aligned with its institutional logics. Instead, markers of success for such a unit might include the successful delivery of service activities to funders, and the satisfaction of the clients and stakeholders affiliated with those HPESU funders. Unfortunately, HPESU leaders do not always realise this incongruity. Furthermore, the leadership of the medical school or hospital may hold differing views on the appropriate weighting of each institutional logic, with the result that HPESU leaders may have to respond to different expectations depending on which member of the leadership they are addressing. 
Working to change the institutional logics underpinning an HPESU is often an arduous and highly political task. The dean, HPESU leaders, clinician-educators, research scientists and other community members all embody and interpret the institutional logics that surround them. Changing those logics involves negotiating across the rules, beliefs and customs each person has internalised and has accepted as representing his or her working culture. As the meanings and values of current practices are supported by the existing configuration of logics, they are not likely to be easily modified. ${ }^{30}$ Our previous research on HPESU administrative leaders as institutional entrepreneurs offers some practical advice on how leaders can shift the institutional logics of their local context. ${ }^{11}$ Successfully engaging as an institutional entrepreneur to change the configurations of these logics in a specific context requires 'the mobilisation and recombination of materials, symbols and people in novel and even artful ways'. ${ }^{30}$ Reconceptualising established logics or mobilising new logics is possible, but the work entailed in achieving these goals should not be underestimated.

We acknowledge that our research is limited by the fact that our data were generated through interviews with HPESU leaders. Other institutional leaders (e.g. medical school deans and hospital leaders) may have different perspectives on the weighting of the institutional logics we identify and may describe other logics as necessary considerations. We plan to extend our investigations to explore how such leaders (e.g. deans, hospital leaders and department chairs) can act as institutional entrepreneurs who negotiate the institutional logics that are instantiated in individual HPESUs. We also intend to explore the perspectives of other agents, such as clinician-educators, as they too may have different understandings of the institutional logics that underpin the HPESU.

Furthermore, as each HPESU is contextually framed by a unique combination of institutional logics, our analysis neither comprehensively nor conclusively identifies all the institutional logics of each HPESU. It also does not investigate how other logics, such as the logic of care,$^{15}$ are often notably absent. Instead, this study explores HPESUs in four different countries to identify common institutional logics and how they are locally and, when relevant, nationally embodied. Finally, this analysis was not informed by data from European, Asian, African or Latin American HPESUs. This is a significant omission, which we are currently addressing by extending our programme of research to the Netherlands, the UK, Sri Lanka, Vietnam and Taiwan.
Institutional logics evolve over time as new logics emerge in a field and as agents engage in individual and collective actions. In any given context, these logics may coexist in relative equality or they may exist in conflict and tension. Over time logics may blend together to construct a brand new institutional logic. We suggest that leaders in HPE (e.g. deans, department chairs, HPESU leaders, etc.) should strive to recognise the logics at play in their context at any given time, and to harness the power of these logics to meet their goals. Perhaps the most challenging demand is the need to stay nimble across institutional logics as logics wax and wane, and to decide which logics should be championed above others.

Contributors: LV is the leader of the health professions education scholarship unit programme of research and led this project. LV led the development of the study design, analysed all study data across all steps of the study design, and prepared all versions of the manuscript (including all major and minor revisions). BO'B contributed to the study design, analysed all of the US data and represented a key analyst in the study. WH contributed to the study design, and critiqued the analysis based on her intimate understanding of the Australian and New Zealand data. OtC, SJD, CvdV, LG, DI, SH-M and $\mathrm{SJH}$ contributed to the study design. All authors contributed to the drafting and critical revision of the paper and approved the final manuscript for publication. All agreed to be accountable for all aspects of the work. Acknowledgements: None.

Funding: None.

Conflicts of interest: None.

Disclaimer: The views expressed herein are those of the authors and do not necessarily reflect those of the United States Department of Defense or other federal agencies. Ethical approval: This study is the fourth in an international programme of research investigating health professions education scholarship units. Each study was approved by the research ethics boards at the relevant institutions (i.e. the University of Western Sydney, the Ottawa Health Science Network and the Uniformed Services University of the Health Sciences). All participants provided informed consent.

\section{REFERENCES}

1 Norman G. Fifty years of medical education research: waves of migration. Med Educ 2011;45 (8):785-91.

2 Lee K, Whelan JS, Tannery NH, Kanter SL, Peters AS. 50 years of publication in the field of medical education. Med Teach 2013;35 (7):591-8.

3 Jaarsma D, Scherpbier A, van der Vleuten C, ten Cate O. Stimulating medical education research in the Netherlands. Med Teach 2013;35 (4):277-81. 
4 van der Vleuten CPM. Medical education research: a vibrant community of research and education practice. Med Educ 2014;48 (8):761-7.

5 Society of Directors of Research in Medical Education. SDRME home page. 2016 http://www.sd rme.org/index.asp. [Accessed 15 January 2015.]

6 Varpio L, Gruppen L, Hu W, O'Brien B, ten Cate O, Humphrey-Murto S, Irby D, van der Vleuten C, Hamstra S, Durning SJ. Working definitions of the roles and an organisational structure in health professions education scholarship: initiating an international conversation. Acad Med 2017;92 (2):205-8.

7 Davis MH, Karunathilake I, Harden RM. AMEE, Education Guide no. 28. The development and role of departments of medical education. Med Teach 2005;27 (8):665-75.

8 Gruppen L. Creating and sustaining centres for medical education research and development. Med Educ 2008;42 (2):121-2.

9 Varpio L, Bidlake E, Humphrey-Murto E, Sutherland S, Hamstra S. Key considerations for the success of medical education research and innovation units in Canada. Adv Health Sci Educ Theory Pract 2014;19 (3):361-77.

$10 \mathrm{Hu}$ WCY, Thistlethwaite JE, Weller J, Gallego G, Monteith J, McColl GJ. 'It was serendipity': a qualitative study of academic careers in medical education. Med Educ 2015;49 (11):1124-36.

11 Varpio L, O'Brien B, Durning SJ, van der Vleuten C, Gruppen L, ten Cate O, Humphrey-Murto S, Irby DM, Hamstra SJ. Health professions education scholarship unit leaders as institutional entrepreneurs. Acad Med 2017. doi: 10.1097/ACM.0000000000001576. [Epub ahead of print.]

12 Thornton PH, Ocasio W, Lounsbury M. The Institutional Logics Perspective: A New Approach to Culture, Structure, and Process. Oxford: Oxford University Press 2012.

13 Thornton PH, Ocasio W. Institutional logics and the historical contingency of power in organisations: executive succession in the higher education publishing industry, 1958-1990. Am J Sociol 1999;105 (3):801-43.

14 Besharaov ML, Smith WK. Multiple organisational logics in organisations: explaining their varied nature and implications. Acad Manage Rev 2014;39 (3):364-81.

15 Dunn MB, Jones C. Institutional logics and institutional pluralism: the contestation of care and science logics in medical education, 1967-2005. Admin Sci Q 2010;55 (1):114-49.

16 Greenwood R, Raynard M, Kodeih F, Micelotta E, Lounsbury M. Institutional complexity and organisational responses. Acad Manage Ann 2011;5 (1):317-71.

17 Greenwood R, Diaz AM, Li SX. The multiplicity of institutional logics and the heterogeneity of organisational responses. Organ Sci 2010;21 (2):521-39.
18 Thornton PH, Ocasto W. Institutional logics. In: Greenwood R, Oliver C, Suddaby R, Sahlin K, eds. The SAGE Handbook of Organizational Institutionalism. Thousand Oaks, CA: Sage Publications 2008;99-129.

19 McPherson CM, Sauder M. Logics in action: managing institutional complexity in a drug court. Admin Sci Q 2013;58 (1):165-96.

20 Booher-Jennings J. Below the bubble: 'educational triage' and the Texas accountability system. Am Educ Res J 2005;42 (2):231-68.

21 Binder A. For love and money: organisations' creative responses to multiple environmental logics. Theory Society 2007;36 (6):547-71.

22 Ad Hoc Committee on Education for Family Practice. Meeting the Challenge of Family Practice. Chicago, IL: American Medical Association 1966.

23 Citizens Commission on Graduate Medical Education. The Graduate Education of Physicians. Chicago, IL: American Medical Association 1966.

24 National Commission on Community Health Services. Health is a Community Affair. Cambridge, MA: Harvard University Press 1966.

25 Hammersley M. Can we Re-Use Qualitative Data Via Secondary Analysis? Notes on Some Terminological and Substantive Issues. Sociol Res Online. 2010;15. http://socresonline.org.uk/15/1/5.html. Accessed January 11, 2017.

26 Kuzzel AJ. Sampling in qualitative inquiry. In: Crabtree F, Miller WL, eds. Doing Qualitative Research, 2nd edn. Thousand Oaks, CA: Sage Publications 1999;33-45.

27 Boyer EL. Scholarship Reconsidered: Priorities of the Professoriate. New York, NY: Jossey-Bass Books 1990.

28 Pache AC, Santos F. Embedded in hybrid contexts: how individuals respond to competing institutional logics. In: Lounsbury M, Boxenbaum E, eds. Institutional Logics in Action, Part B. Bingley: Emerald Group Publishing 2013;3-35.

29 Lok J. Institutional logics as identity projects. Acad Manag J 2010;53 (6):1305-35.

30 Hardy C, Maguire S. Institutional entrepreneurship. In: Greenwood R, Oliver C, Suddaby R, Sahlin K, eds. The SAGE Handbook of Organizational Institutionalism. Thousand Oaks, CA: Sage Publications 2008;198-217.

\section{SUPPORTING INFORMATION}

Additional Supporting Information may be found in the online version of this article:

Appendix S1. Abbreviated protocol for semistructured interviews with US health professions education scholarship unit leaders.

Received 17 October 2016; editorial comments to author 7 December 2016, accepted for publication 6 February 2017 